\title{
Amplitude-modulated, Cosine PE and WP Pulses: Theory and Applicability
}

\author{
Ioan E. LAGER ${ }^{1}$, Martin STUMPF ${ }^{2}$ \\ ${ }^{1}$ Faculty EEMCS, Delft University of Technology, Mekelweg 4, 2628 CD Delft, the Netherlands \\ ${ }^{2}$ Department of Radioelectronics, Brno University of Technology, 61600 Brno, Czech Republic \\ i.e.lager@tudelft.nl, martin.stumpf@centrum.cz \\ Submitted February 12, 2021 / Accepted March 2, 2021
}

\begin{abstract}
The amplitude-modulated, cosine powerexponential (PE) and windowed-power (WP) pulses are discussed, by insisting on their time-domain normalization. Illustrative examples of signatures and their correspondent frequency-domain behavior are given. These examples compellingly demonstrate the possibility to replace non-causal pulses of prevalent use by causal, or even time-windowed, pulses with closely resembling signatures.
\end{abstract}

\section{Keywords}

Causality, time-domain analysis, pulse generation

\section{Introduction}

Pulse shape (discrimination) is a topic of broad scientific interest, ranging from particle physics [1-3] (where it allows distinguishing between signals from neutrons and gamma rays) to ubiquitous technologies such as radar and (wireless) communication. When zooming in on wireless communications, the major challenge is responding to the exploding needs for data transfer, with ultra-wideband (UWB) technology being one of the most viable candidates for securing ultra-high data rates in conjunction with manageable power requirements [4]. Reliable and fast data transfer critically depends on preserving the pulse shape, with [5-7] focussing on the UWB figures of merit that are of relevance to this end. One of the important roadblocks in achieving high throughput and low bit errors is the intersymbol interference (ISI) [8, p. 43]. Here, dispersion and pulse ringing are the two channel-related causes of ISI, with ringing putting a cap on the pulse repetition rate and, thus, on the baud-rate. As a result, important efforts are invested in mitigating the pulse ringing, as evidenced by the (technological) approaches advocated in [9-12].
At this point, it is noted that all explorations in [5-7,9-12] rely on using (time-differentiated) Gaussian pulses, a patently non-causal signature ${ }^{1}$. Recently, [13] highlighted the need to ensure causality in electromagnetic (EM) investigations. That publication catalogued a number of strictly causal pulses, two of them having ringing shapes.

This survey will examine the power-exponential (PE) and windowed-power (WP) amplitude-modulated, cosine pulses. While the PE variant was introduced in [14], the WP variant was not yet presented. A particular emphasis will be placed on the normalization of these pulses, that methodology being potentially applicable to the design of ringing shapes using different apodizations $[15$, p. 55]. Typical signatures and spectra of the advocated pulses will be provided. The pulses will then be shown to have the ability to accurately replicate time-domain (TD) signatures of pulses generated by existing circuitry, thus allowing the incorporation of causal, analytic copies of the relevant signals in (EM) models or within design strategies concerning complex systems.

\section{Definition of Pulses}

The PE and WP unipolar prototype pulses were elaborately discussed in [14], [16], their TD expressions being

$$
\operatorname{PE}\left(v, t_{\mathrm{r}}, t\right)=t^{\prime v} \exp \left[-v\left(t^{\prime}-1\right)\right] H\left(t^{\prime}\right)
$$

and

$$
\mathrm{WP}\left(v, t_{\mathrm{r}}, t\right)=t^{\prime v}\left(2-t^{\prime}\right)^{v} H\left(t^{\prime}\right) H\left(2-t^{\prime}\right)
$$

respectively. In these expressions $v \geqslant 1$ is the pulse raising power, $t_{\mathrm{r}}$ is the pulse rise-time ${ }^{2}, t$ is the time coordinate, $t^{\prime}=t / t_{\mathrm{r}}$ is the normalized time coordinate and $H(\cdot)$ is the Heaviside step function. For practical reasons, $v$ is taken to assume only integer values. Furthermore, taking $v \geqslant 3$ ensures the continuity of the pulses and their first two time derivatives at $t^{\prime}=0$, and at $t^{\prime}=2$ for WP (see [14], [16]).

\footnotetext{
${ }^{1}$ Interestingly, [6] already noted the non-causality of the employed signature, but surmised that the inevitable truncation errors do not significantly impact on the system performance.

${ }^{2}$ The pulse rise-time $t_{\mathrm{r}}>0$ is defined as the time between the unipolar pulse's onset and the instant when it reaches its maximum. In the case of the time-differentiated versions of the PE and WP pulses, $t_{\mathrm{r}}$ also represents the zero-crossing time of the relevant pulses.
} 

is [14]

The frequency-domain (FD) behavior of the PE pulse

$$
\widehat{\mathrm{PE}}\left(v, t_{\mathrm{r}}, \omega\right)=t_{\mathrm{r}} \frac{\Gamma(v+1) \exp (v)}{\left(\mathrm{j} \omega t_{\mathrm{r}}+v\right)^{v+1}}
$$

in which $\omega$ is the angular frequency $(\omega=2 \pi f$, with $f$ being the frequency), and $\Gamma(\cdot)$ denotes the Euler gamma function since $v$ is integer, $\Gamma(v)=(v-1)$ !. The FD behavior of the $\mathrm{PE}$ pulse is [16]

$$
\widehat{\mathrm{WP}}\left(v, t_{\mathrm{r}}, \omega\right)=\exp \left(-\mathrm{j} \omega t_{\mathrm{r}}\right) t_{\mathrm{r}} 2^{v} v ! \sqrt{2 \pi} \frac{J_{v+1 / 2}\left(\omega t_{\mathrm{r}}\right)}{\left(\omega t_{\mathrm{r}}\right)^{v+1 / 2}}
$$

where $J_{n+1 / 2}$ is the Bessel function of the first kind and fractional order [17, Sec. 10.1]. Note that $|\widehat{\mathrm{PE}}(\omega)|=|\widehat{\mathrm{PE}}(-\omega)|$ and $|\widehat{\mathrm{WP}}(\omega)|=|\widehat{\mathrm{WP}}(-\omega)|$ since the pulses are real functions.

The PE was used in [14] for constructing the ringing cosine $\mathrm{PE}_{\mathrm{cos}}$ pulse via the expression

$$
\mathrm{PE}_{\mathrm{cos}}\left(v, t_{\mathrm{r}}, \kappa_{\mathrm{w}}, t\right)=\operatorname{PE}\left(v, t_{\mathrm{r}}, t\right) \cos \left(2 \pi \tilde{\omega}_{\mathrm{w}} t^{\prime}\right)
$$

in which $\kappa_{\mathrm{W}}$ is a 'wiggle factor' that, for a harmonic carrier of frequency $f_{0}$, counts the carrier cycles per $t_{\mathrm{r}}$ as $\kappa_{\mathrm{w}}=t_{\mathrm{r}} f_{0}$, and $\tilde{\omega}_{\mathrm{w}}=2 \pi \kappa_{\mathrm{w}}$. By using the same strategy, a WP ringing cosine pulse is defined via

$$
\mathrm{WP}_{\mathrm{cos}}\left(v, t_{\mathrm{r}}, \kappa_{\mathrm{w}}, t\right)=\mathrm{WP}\left(v, t_{\mathrm{r}}, t\right) \cos \left(\tilde{\omega}_{\mathrm{w}} t^{\prime}\right) .
$$

The frequency-domain (FD) behavior of $\mathrm{PE}_{\mathrm{cos}}$ and $\mathrm{WP}_{\mathrm{cos}}$ can be easily derived, the relevant expresions being

$$
\begin{aligned}
& \widehat{\mathrm{PE}}_{\mathrm{cos}}\left(v, t_{\mathrm{r}}, \kappa_{\mathrm{W}}, \omega\right) \\
& =\frac{1}{2}\left[\widehat{\mathrm{PE}}\left(v, t_{\mathrm{r}}, \omega-\omega_{0}\right)+\widehat{\mathrm{PE}}\left(v, t_{\mathrm{r}}, \omega+\omega_{0}\right)\right]
\end{aligned}
$$

and

$$
\begin{aligned}
& \widehat{\mathrm{WP}}_{\mathrm{cos}}\left(v, t_{\mathrm{r}}, \kappa_{\mathrm{w}}, \omega\right) \\
& =\frac{1}{2}\left[\widehat{\mathrm{WP}}\left(v, t_{\mathrm{r}}, \omega-\omega_{0}\right)+\widehat{\mathrm{WP}}\left(v, t_{\mathrm{r}}, \omega+\omega_{0}\right)\right]
\end{aligned}
$$

in which $\omega_{0}=2 \pi f_{0}=2 \pi \kappa_{\mathrm{w}} / t_{\mathrm{r}}$.

For studying (electronic) circuits in which no net electric charge is transported (as the ones in antenna engineering), time-differentiated versions of the thus far introduced pulses are needed. The relevant pulses are denoted as $\partial_{t} \mathrm{PE}$, $\partial_{t} \mathrm{WP}, \partial_{t} \mathrm{PE}_{\mathrm{cos}}$ and $\partial_{t} \mathrm{WP}_{\mathrm{cos}}$. Their TD and FD expressions follow from the corresponding, original expressions (the FD behavior requiring simply a multiplication by $\mathrm{j} \omega$ ).

\section{Normalization of Pulse TD Signatures}

The normalization of the $\partial_{t} \mathrm{PE}$ and $\partial_{t} \mathrm{WP}$ was elaborately discussed in [14], [16] and will not be repeated here.
When turning to the ringing cosine pulses, it is observed that the normalization could be applied to the pulse itself. However, such a pulse normalization does not lend itself to an analytic handling and, thus, is not considered here. Instead, our approach is to normalize the pulses' envelopes. By that standard, $\mathrm{PE}_{\mathrm{cos}}$ and $\mathrm{WP}_{\mathrm{cos}}$ are readily normalized, since the expressions (1) and (2) are implicitly normalized.

The time-differentiated, ringing cosine pulses need a more careful examination. For simplicity, $H(\cdot)$ will be omitted from the derived expressions, its effect being implicitly accounted for. The explicit mentioning of $t_{\mathrm{r}}$ and $t$ as arguments of PE and WP will be also omitted.

By time-differentiating (5), it follows that

$$
\begin{aligned}
& \partial_{t} \mathrm{PE}_{\mathrm{cos}}\left(v, t_{\mathrm{r}}, \kappa_{\mathrm{W}}, t\right)=\partial_{t}\left[\mathrm{PE}(v) \cos \left(\tilde{\omega}_{\mathrm{w}} t^{\prime}\right)\right] \\
& =\omega_{0} \mathrm{PE}(v)\left[\mathscr{K}_{1}\left(t^{\prime}\right) \cos \left(\tilde{\omega}_{\mathrm{w}} t^{\prime}\right)-\sin \left(\tilde{\omega}_{\mathrm{w}} t^{\prime}\right)\right]
\end{aligned}
$$

in which

$$
\mathscr{K}_{1}\left(t^{\prime}\right)=\beta_{1} \frac{1-t^{\prime}}{t^{\prime}}=\frac{1}{\tan \left[\alpha_{1}\left(t^{\prime}\right)\right]} \quad \text { for } t^{\prime}>0
$$

with $\beta_{1}=1 / \tilde{\omega}_{\mathrm{w}}$. By substituting (10) in (9), and using some standard trigonometric identities it is found that

$$
\partial_{t} \mathrm{PE}_{\mathrm{cos}}\left(v, t_{\mathrm{r}}, \kappa_{\mathrm{w}}, t\right)=\omega_{0} \mathscr{F}_{1}\left(t^{\prime}\right) \cos \left[\tilde{\omega}_{\mathrm{w}} t^{\prime}+\alpha_{1}\left(t^{\prime}\right)\right]
$$

in which

$$
\begin{aligned}
& \mathscr{F}_{1}\left(t^{\prime}\right)=\frac{\operatorname{PE}(v)}{\sin \left[\alpha_{1}\left(t^{\prime}\right)\right]} \\
& =\operatorname{PE}(v-1) \exp \left(1-t^{\prime}\right)\left[t^{\prime 2}+\beta_{1}^{2}\left(1-t^{\prime}\right)^{2}\right]^{1 / 2} .
\end{aligned}
$$

Since $v \geqslant 3, \operatorname{PE}(v-1)$ is well-defined. The function $\mathscr{F}_{1}\left(t^{\prime}\right)$ is finite, with $\lim _{t^{\prime} \downarrow 0} \mathscr{F}_{1}\left(t^{\prime}\right)=0, \mathscr{F}_{1}(1)=1$, and $\lim _{t^{\prime} \rightarrow \infty} \mathscr{F}_{1}\left(t^{\prime}\right)=0$. The shape of $\mathscr{F}_{1}\left(t^{\prime}\right)$ is dictated by $\operatorname{PE}(v-1)$ that peaks at 1 for $t^{\prime}=1$. It can now be concluded that the envelope of the $\partial_{t} \mathrm{PE}_{\mathrm{cos}}$ pulse is normalized by multiplying the time derivative by $1 / \omega_{0}=\left(2 \pi f_{0}\right)^{-1}$, a fact already stated in [14, Eq. (15)].

By time-differentiating (6), it follows that

$$
\begin{aligned}
& \partial_{t} \mathrm{WP}_{\cos }\left(v, t_{\mathrm{r}}, \kappa_{\mathrm{W}}, t\right)=\partial_{t}\left[\mathrm{WP}(v) \cos \left(\tilde{\omega}_{\mathrm{w}} t^{\prime}\right)\right] \\
& =\omega_{0} \mathrm{WP}(v)\left[\mathscr{K}_{2}\left(t^{\prime}\right) \cos \left(\tilde{\omega}_{\mathrm{w}} t^{\prime}\right)-\sin \left(\tilde{\omega}_{\mathrm{w}} t^{\prime}\right)\right]
\end{aligned}
$$

in which

$$
\mathscr{K}_{2}\left(t^{\prime}\right)=\beta_{2} \frac{1-t^{\prime}}{t^{\prime}\left(2-t^{\prime}\right)}=\tan \left[\alpha_{2}\left(t^{\prime}\right)\right] \quad \text { for } 0<t^{\prime}<2
$$

with $\beta_{2}=v /\left(\pi \kappa_{\mathrm{w}}\right)$. By substituting (14) in (13), and using some standard trigonometric identities it is found that

$$
\partial_{t} \mathrm{WP}_{\cos }\left(v, t_{\mathrm{r}}, \kappa_{\mathrm{W}}, t\right)=\omega_{0} \mathscr{F}_{2}\left(t^{\prime}\right) \cos \left[\tilde{\omega}_{\mathrm{W}} t^{\prime}+\alpha_{2}\left(t^{\prime}\right)\right]
$$

in which

$$
\begin{aligned}
& \mathscr{F}_{2}\left(t^{\prime}\right)=\frac{\mathrm{WP}(v)}{\sin \left[\alpha_{2}\left(t^{\prime}\right)\right]} \\
& =\mathrm{WP}(v-1)\left[t^{\prime 4}-4 t^{\prime 3}+\left(4+\beta_{2}\right) t^{\prime 2}-2 \beta_{2}^{2} t^{\prime}+\beta_{2}^{2}\right]^{1 / 2} .
\end{aligned}
$$


Since $v \geqslant 3, \operatorname{WP}(v-1)$ is well-defined. The multiplier of $\mathrm{WP}(v-1)$ is a positive, smooth function that is symmetric with respect to $t^{\prime}=1$ and takes the values $\beta_{2}$ for $t^{\prime}=0$ and $t^{\prime}=2$, and 1 for $t^{\prime}=1$. The shape of $\mathscr{F}_{2}\left(t^{\prime}\right)$ is dictated by $\mathrm{WP}(v-1)$ that peaks at 1 for $t^{\prime}=1$. It can now be concluded that the envelope of the $\partial_{t} \mathrm{WP}_{\cos }$ pulse is also normalized by multiplying the time derivative by $1 / \omega_{0}=\left(2 \pi f_{0}\right)^{-1}$.

\section{Numerical Experiments}

The practical utility of the introduced ringing signals is henceforth argued by examining some typical signatures and exploring their ability to replicate signals generated by existing circuitry.

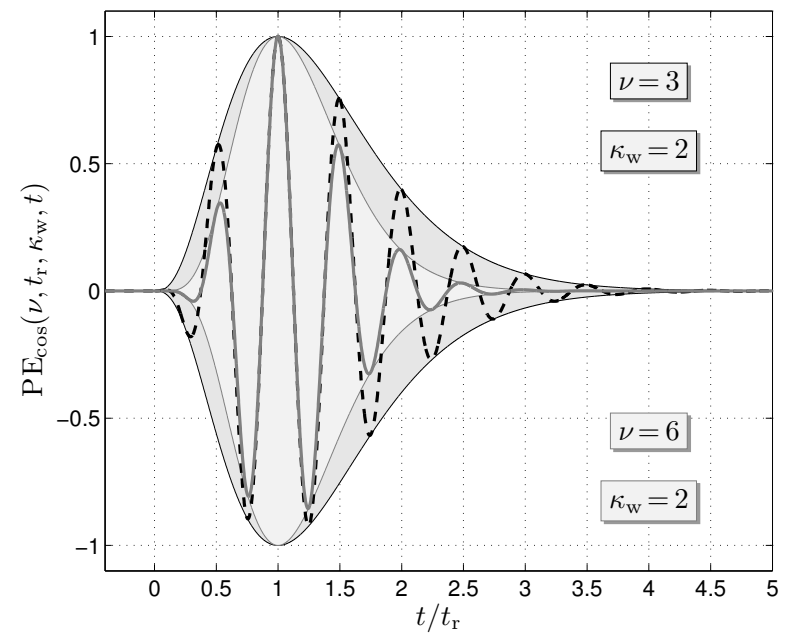

(a) Signatures

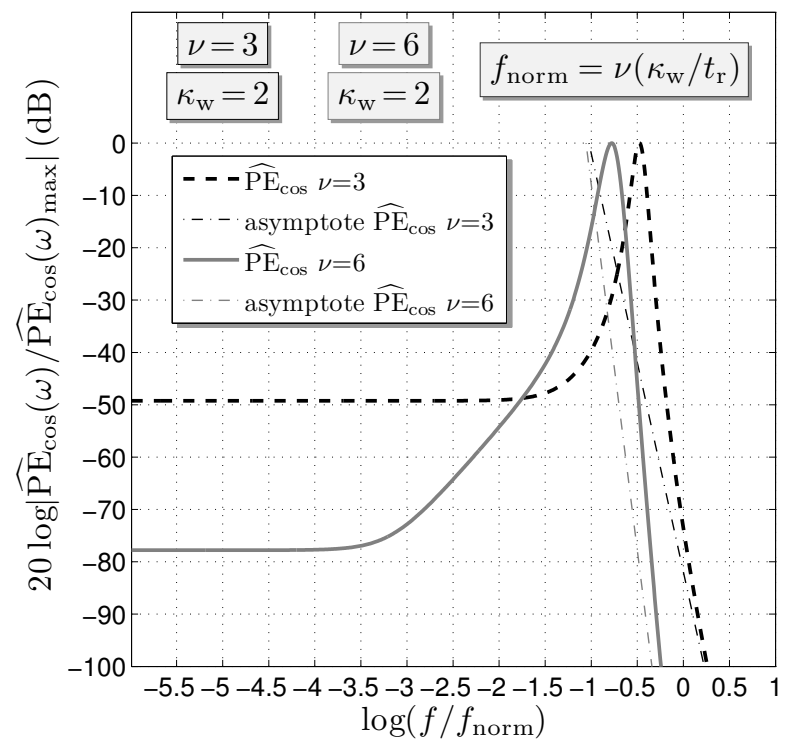

(b) Spectral behavior

Fig. 1. $\mathrm{PE}_{\mathrm{cos}}$ pulse with $v=3$ (dashed line) and $v=6$ (solid line), $t_{\mathrm{r}}=1$ and $\kappa_{\mathrm{W}}=2$. The solid patches in (a) represent the pulse envelopes, with the lighter patch corresponding to $v=6$. The asymptotes of the spectral contents are shown in (b).

\subsection{Examples}

Some typical signatures of ringing cosine pulses are now given. For each pulse, the signature, the envelope and the spectral behavior will be given.

PE-type signals are firstly discussed. Figure 1 shows two $\mathrm{PE}_{\mathrm{cos}}$ pulses with $t_{\mathrm{r}}=1, \kappa_{\mathrm{W}}=2$, and $v$ assuming the values 3 and 6 , respectively. The TD plots illustrate the effect of $v$ on the pulse's shape. The spectral behavior has a nonzero DC value and peaks, as expected, at $f_{0}{ }^{3}$. The $\partial_{t} \mathrm{PE}_{\mathrm{cos}}$ performance is demonstrated in Fig. 2 for the same pulse parameters as those in Fig. 1. The differences in the TD signatures are small and they manifest primarily in the beginning of the pulse.

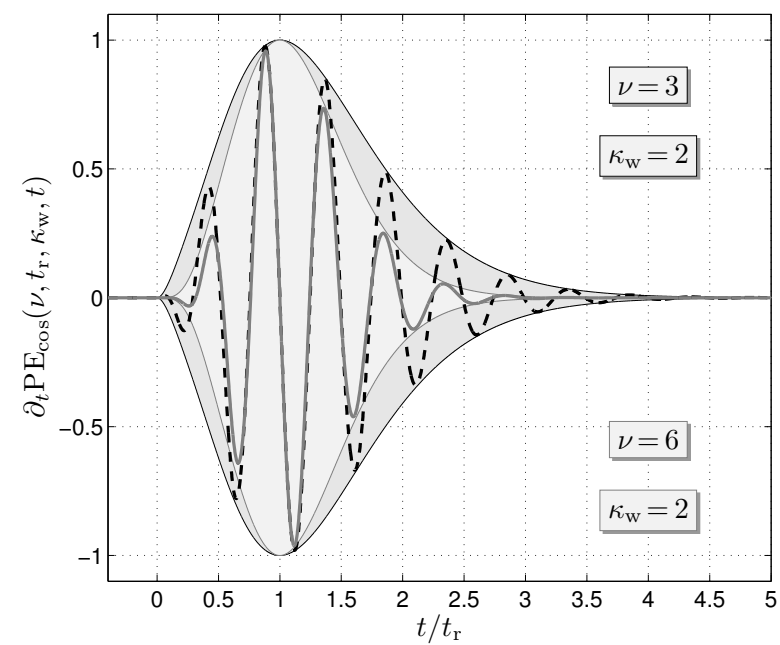

(a) Signatures

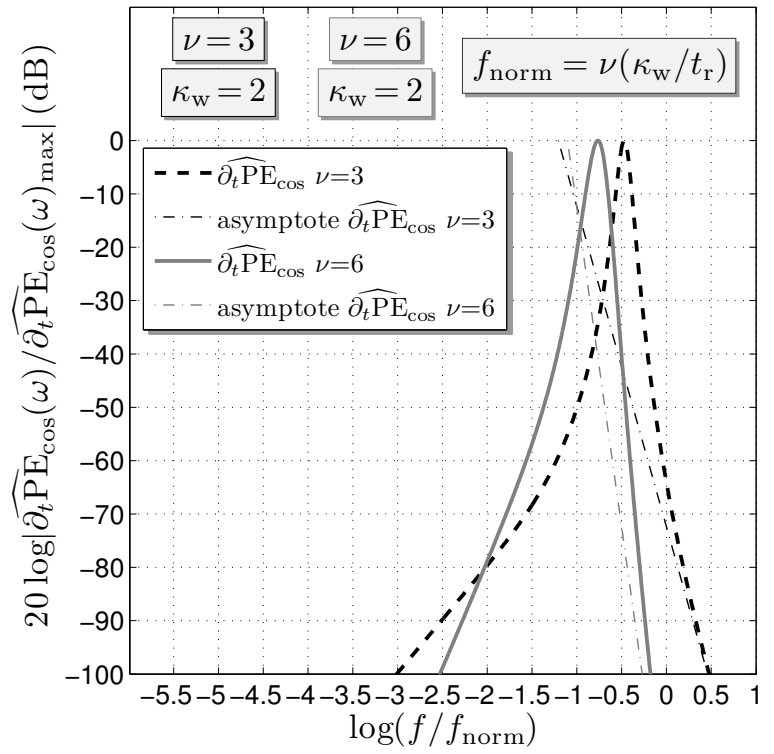

(b) Spectral behavior

Fig. 2. $\partial_{t} \mathrm{PE}_{\mathrm{cos}}$ pulse with $v=3$ (dashed line) and $v=6$ (solid line), $t_{\mathrm{r}}=1$ and $\kappa_{\mathrm{W}}=2$. The solid patches in (a) represent the pulse envelopes, with the lighter patch corresponding to $v=6$. The asymptotes of the spectral contents are shown in (b).

${ }^{3}$ The definition of $f_{\text {norm }}$ entails that $f_{0} / f_{\text {norm }}=1 / v$, implying that the spectral diagrams' peaks correspond to the abscissas -0.47 and -0.78 for $v=3$ and 6 , respectively. 
The spectral behavior resembles that of the $\mathrm{PE}_{\mathrm{cos}}$ above $f_{0}$ but, evidently, has no DC component. From these signatures it can be inferred that the PE-modulated pulses are opportune for modeling ringing signatures with a sharp increase and a long tail, with $\partial_{t} \mathrm{PE}_{\mathrm{cos}}$ having the capability to reflect the situations when no net electric charge is transported in a circuit.

Upon now turning to WP-type signals, Figure 3 shows two $\mathrm{WP}_{\text {cos }}$ pulses with $t_{\mathrm{r}}=1, \kappa_{\mathrm{W}}=4$, and $v=4,8$, respectively. Again, the effect of $v$ on the pulse's shape is noticeable. The spectral behavior bears some overall resemblance

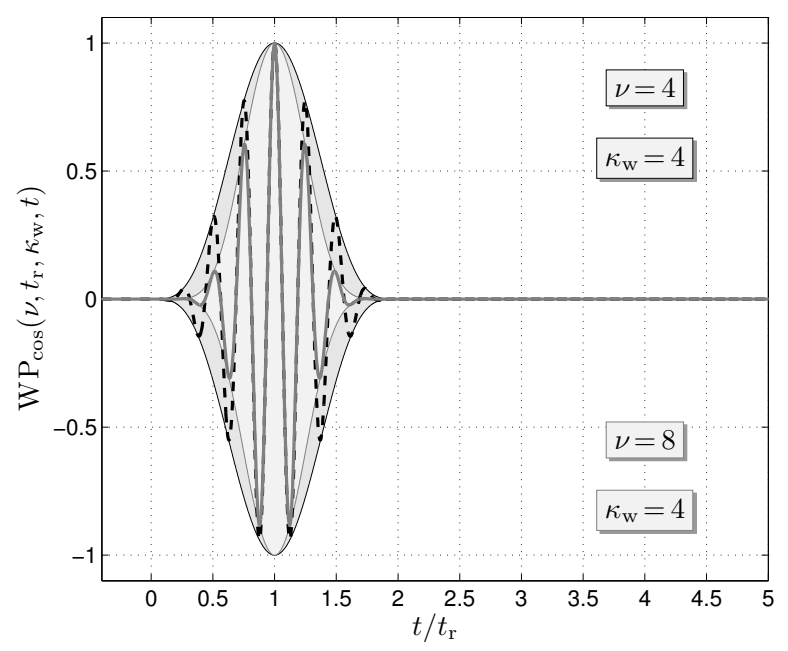

(a) Signatures

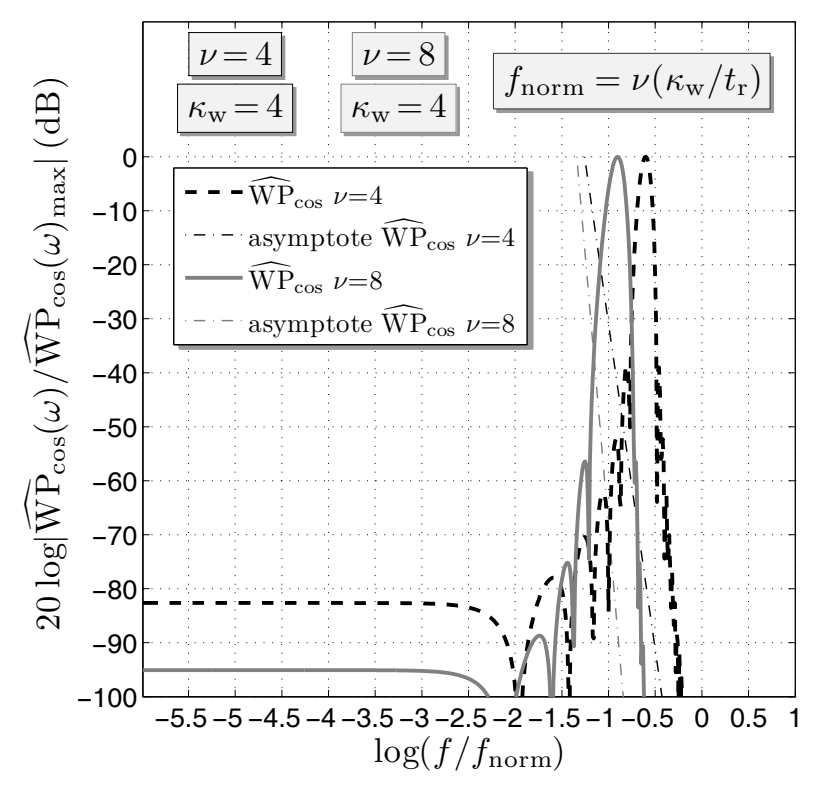

(b) Spectral behavior

Fig. 3. $\mathrm{WP}_{\cos }$ pulse with $v=4$ (dashed line) and $v=8$ (solid line), $t_{\mathrm{r}}=1$ and $\kappa_{\mathrm{w}}=4$. The solid patches in (a) represent the pulse envelopes, with the lighter patch corresponding to $v=8$. The asymptotes of the spectral contents are shown in (b). with that in Fig. 1, but the lobe around $f_{0}$ is narrower and the DC level is lower, which concurs with the very low spectral leakage of the WP prototype proven in [16] (to a lesser degree, these effects are also referable to the higher $v$ in these experiments). The corresponding $\partial_{t} \mathrm{WP}_{\cos }$ performance following from using the same pulse parameters is exemplified in Fig. 4. The same observations as in the case of the PE-type signals can be made. The presented signatures recommend the WP-type pulses for situations requiring a time-windowed harmonic function. A typical such application can be found in the field of timed antennas [18] (the advantages of using the WP apodization were elaborately explained in [13], [16]).

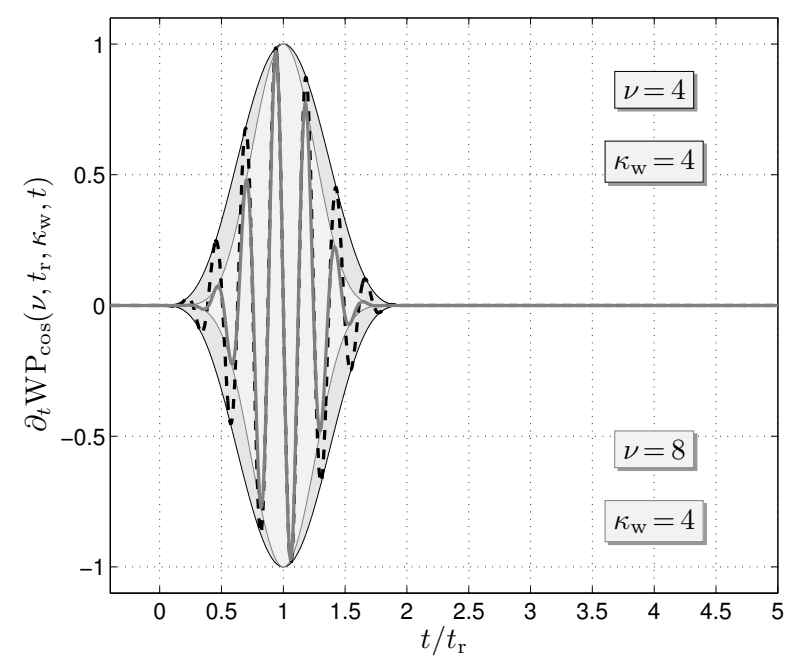

(a) Signatures

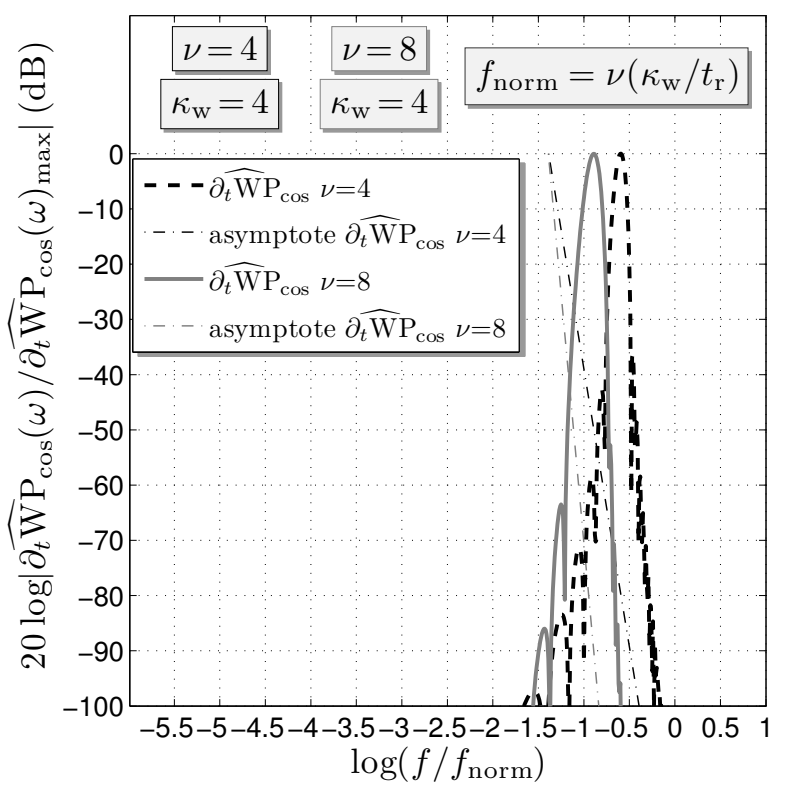

(b) Spectral behavior

Fig. 4. $\partial_{t} \mathrm{WP}_{\cos }$ pulse with $v=4$ (dashed line) and $v=8$ (solid line), $t_{\mathrm{r}}=1$ and $\kappa_{\mathrm{W}}=4$. The solid patches in (a) represent the pulse envelopes, with the lighter patch corresponding to $v=8$. The asymptotes of the spectral contents are shown in (b). 


\subsection{Replication}

A second investigation concerns the ability of the advocated pulses to mimic signatures that are effectively generated by existing circuitry. The signatures in $[11$, Fig. $2 c, d]$ are selected to this end - note that those signatures were constructed by starting from non-causal Gaussian-type pulses. Hereafter, original signals will be generically denoted as $S_{\text {orig }}(t)$, while $S_{\text {repl }}(t)$ will stand for the constructed replica. The replication accuracy is assessed based on an energy percentage replication error that is calculated with the expression

$$
\operatorname{Err}_{\%}=\frac{\int_{\mathcal{T}}\left[S_{\text {orig }}(t)-S_{\text {repl }}(t)\right]^{2} \mathrm{~d} t}{\int_{\mathcal{T}}\left[S_{\text {orig }}^{2}(t)\right] \mathrm{d} t} 100
$$

in which $\mathcal{T}$ is the interval between the earliest among the onsets of $S_{\text {orig }}$ and $S_{\text {repl }}$ and the end $S_{\text {orig. }}$.

The original signals in $[11$, Fig. $2 c, d]$ are shown in Figs. 5 and 6 , respectively ${ }^{4}$. Since both signatures feature a long tail, a PE-type replication is selected based on the observations in Sec. 4.1. The mimicking was effectuated via a heuristic approach ${ }^{5}$ employing a grid-search over $v, t_{\mathrm{r}}$ and $f_{0}$ (and, implicitly, $\kappa_{\mathrm{w}}$ ) that aimed at minimizing the error in (17). This procedure was implemented in Matlab ${ }^{\circledR}$. Since the goal of this exercise was to obtain a good match and it was intended as a singular experiment, providing information on computational effectiveness is deemed irrelevant.

To begin with, initial guesses were made for $t_{\mathrm{r}}$ and $f_{0}$ by measuring some features of $S_{\text {orig. }}$. It was then observed that the signature in $[11$, Fig. 2c] lends itself to a replication via a PE pulse with a low $v$, whereas that in [11, Fig. 2d] lends itself to a replication via a $\partial_{t} \mathrm{PE}$ pulse with a high $v$. Based on these tests, a PE with $v=4$ and a $\partial_{t} \mathrm{PE}$ with $v=24$ were selected. The next phase was a full grid search on ranges of $t_{\mathrm{r}}$ and $f_{0}$ values centered around the initial guesses. The constructed pulses' magnitudes $\left(V_{\mathrm{m}}\right)$ were determined by matching the maxima in the originals and the replicas. While these searches did yield some optimal values, the entailed Err\% was considered too large. In order to improve the accuracy, the replicas' onsets were allowed to move by some (small) $t_{\text {shift }}$ time shifts with respect to the onsets in $S_{\text {orig. }}$ This choice entailed a third grid-search parameter that eventually, led to extremely satisfactory results. Specifically, the following replicas were constructed:

1. For the signature in [11, Fig. 2c]: A PE pulse with $V_{\mathrm{m}}=3.94 \mathrm{~V}, v=4, t_{\mathrm{r}}=0.52 \mathrm{~ns}, f_{0}=1.67 \mathrm{GHz}$ $\left(\kappa_{\mathrm{w}}=0.87\right)$; the replica is delayed by $t_{\text {shift }}=119$ ps. The replication error amounts to Err $_{\%}=5.2 \%$. The constructed $S_{\text {repl }}$ is superimposed on $S_{\text {orig }}$ in Fig. 5.
2. For the signature in [11, Fig. 2d]: A $\partial_{t}$ PE pulse with $V_{\mathrm{m}}=2.35 \mathrm{~V}, v=24, t_{\mathrm{r}}=1.06 \mathrm{~ns}, f_{0}=1.84 \mathrm{GHz}$ $\left(\kappa_{\mathrm{w}}=1.95\right)$; the replica is delayed by $t_{\text {shift }}=7 \mathrm{ps}$. The replication error amounts to $\operatorname{Err}_{\%}=2.2 \%$. The constructed $S_{\text {repl }}$ is superimposed on $S_{\text {orig }}$ in Fig. 6.

Another example of mimicking a measured signal via a $\partial_{t} \mathrm{PE}$ signature can be found in [14, Sec. VI.A]. These experiments cogently prove the possibility to obtain extremely accurate causal, analytic copies of physically generated signals - these functions can then be used in models or within design strategies concerning complex systems.

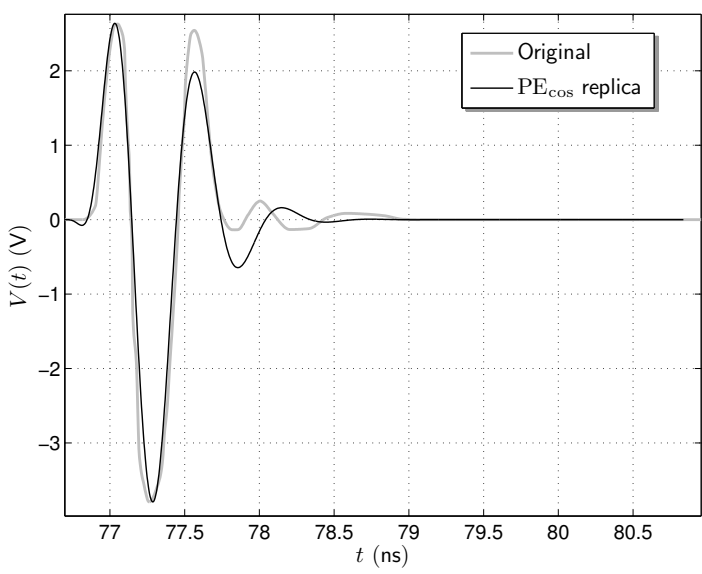

Fig. 5. Replication of the signature in [11, Fig. 2c] via a $\mathrm{PE}_{\mathrm{cos}}$ pulse with parameters: $V_{\mathrm{m}}=3.94 \mathrm{~V}, v=4, t_{\mathrm{r}}=0.52 \mathrm{~ns}$, $f_{0}=1.67 \mathrm{GHz}\left(\kappa_{\mathrm{W}}=0.87\right)$. The replica's onset succedes the original signal's onset by $t_{\text {shift }}=119$ ps. The replication error amounts to Err\% $=5.2 \%$.

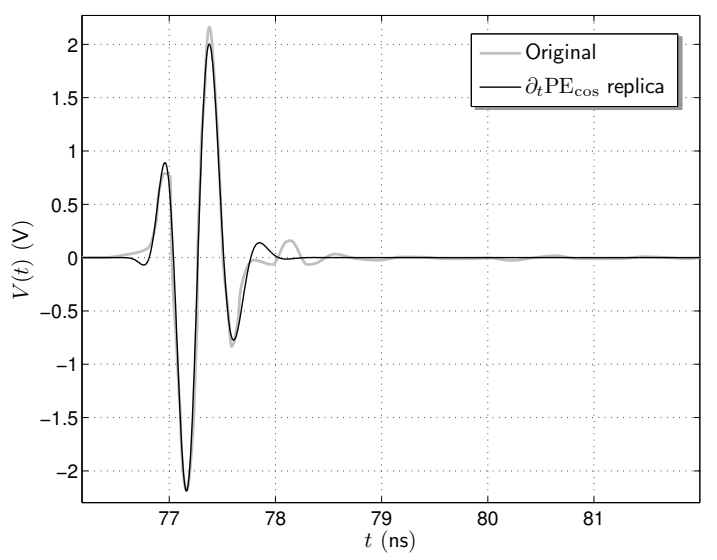

Fig. 6. Replication of the signature in $\left[11\right.$, Fig. 2d] via a $\partial_{t} \mathrm{PE}_{\mathrm{cos}}$ pulse with parameters: $V_{\mathrm{m}}=2.35 \mathrm{~V}, v=24$, $t_{\mathrm{r}}=1.06 \mathrm{~ns}, f_{0}=1.84 \mathrm{GHz}\left(\kappa_{\mathrm{W}}=1.95\right)$. The replica's onset precedes the original signal's onset by $t_{\text {shift }}=7 \mathrm{ps}$. The replication error amounts to Err $_{\%}=2.2 \%$.

${ }^{4}$ The signatures in Figs. 5 and 6 intentionally exclude some small oscillations preceding the onset that were present in the signals given in [11] (those oscillations do not actually pertain to the intended pulses and are deemed as irrelevant for replication purposes).

${ }^{5}$ More sophisticated strategies could also have been employed. Nonetheless, this experiment aimed at demonstrating the similarity between the original signatures and some of the advocated pulses, and not at obtaining an optimal replication of it. The selected heuristic approach serves this purpose. 


\section{Conclusions}

Two types of causal, normalized, ringing pulses, namely the amplitude-modulated, cosine power-exponential (PE) and windowed-power (WP) pulses were discussed. Their definition makes use of a small set of intuitive parameters. The pulses' ease of use is augmented by their Fourier transform being analytical. Some typical signatures and spectral diagrams were presented, these illustrating the pulses' ability to mimic either ringing signatures with a sharp increase and a long tail (PE) or time-windowed harmonic functions (WP). A combination of PE and time-differentiated PE pulses was shown to accurately replicate time-domain behavior of pulses generated by existing circuitry, thus creating the conditions for incorporating realistic signatures in complex models or design frameworks via causal, analytic signatures.

\section{References}

[1] VARGA, L. Pulse shape discrimination. Nuclear Instruments and Methods, 1961, vol. 14, p. 24-32. DOI: 10.1016/0029-554X(61)90047-7

[2] ROUSH, M.L., WILSON, M. A., HORNYAK, W.F. Pulse shape discrimination. Nuclear Instruments and Methods, 1964, vol. 31, p. 112-124. DOI: 10.1016/0029-554X(64)90333-7

[3] LANGEVELD, W. G. J., KING, M. J., KWONG, J., et al. Pulse shape discrimination algorithms, figures of merit, and Gamma-rejection for liquid and solid scintillators. IEEE Transactions on Nuclear Science, 2017, vol. 64, no. 7, p. 1801-1809. DOI: 10.1109/TNS.2017.2681654

[4] LAGER, I.E., STASZEWSKI, R.B., SMOLDERS, A.B., et al. Ultra-high data-rate wireless transfer in a saturated spectrum - new paradigms. In Proceedings of the 44th European Microwave Conference (EuMC). Rome (Italy), 2014, p. 917-920. DOI: 10.1109/EuMC.2014.6986585

[5] MCLEAN, J. S. FOLTZ, F., SUTTON, R. Pattern descriptors for UWB antennas. IEEE Transactions on Antennas and Propagation, 2005, vol. 53, no. 1, p. 553-2215. DOI: 10.1109/TAP.2004.838757

[6] KWON, D.-H. Effect of antenna gain and group delay variations on pulse-preserving capabilities of ultrawideband antennas. IEEE Transactions on Antennas and Propagation, 2006, vol. 54, no. 8, p. 2208-2215. DOI: 10.1109/TAP.2006.879189

[7] RAMBABU, K., TAN, A. E., CHAN, K. K., et al. Estimation of antenna effect on ultra-wideband pulse shape in transmission and reception IEEE Transactions on Electromagnetic Compatibility, 2009, vol. 51, no. 3, p. 604-610. DOI: 10.1109/TEMC.2009.2023364

[8] ZHANG, X., LARSON, L. E., ASBECK, P. M. Design of Linear RF Outphasing Power Amplifiers. Norwood (USA, MA): Artech House, 2003. ISBN: 9781580536127

[9] HAN, J. NGUYEN, C. A new ultra-wideband, ultra-short monocycle pulse generator with reduced ringing. IEEE Microwave and Wireless Components Letters, 2002, vol. 12, no. 6, p. 206-208. DOI: 10.1109/LMWC.2002.1009996

[10] XIA, T., VENKATACHALAM, A. S., HUSTON, D. A highperformance low-ringing ultrawideband monocycle pulse generator. IEEE Transactions on Instrumentation and Measurement, 2012, vol. 61, no. 1, p. 261-266. DOI: 10.1109/TIM.2011.2161022
[11] VALIZADE, A., REZAEI, P., OROUJI, A. A. A design of UWB reconfigurable pulse transmitter with pulse shape modulation. Microwave and Optical Technology Letters, 2016, vol. 58, no. 9, p. 2221-227. DOI: $10.1002 / \mathrm{mop} .30016$

[12] SHARMA, A., SHARMA, S.K. Spectral efficient pulse shape design for UWB communication with reduced ringing effect and performance evaluation for IEEE 802.15.4a channel. Wireless Networks, 2019, vol. 25, no. 5, p. 2723-2740. DOI: $10.1007 / \mathrm{s} 11276-019-01989-6$

[13] LAGER, I. E. Causal excitation in antenna simulations. Radioengineering, 2021, vol. 30, no. 1, p. 1-9. DOI: 10.13164/re.2021.0001

[14] LAGER, I.E., DE HOOP, A.T., KIKKAWA, T. Model pulses for performance prediction of digital microelectronic systems. IEEE Transactions on Components, Packaging, and Manufacturing Technology, 2012, vol. 2, no. 11, p. 1859-1870. DOI: 10.1109/TCPMT.2012.2216266

[15] WEISSTEIN, E.W. CRC Concise Encyclopedia of Mathematics. Boca Raton (USA, FL): CRC Press LLC, 1999. ISBN: 9780849319457

[16] LAGER, I. E., VAN BERKEL S. L. Finite temporal support pulses for EM excitation. IEEE Antennas and Wireless Propagation Letters, 2017, vol. 16, p. 1659-1662. DOI: 10.1109/LAWP.2017.2662205

[17] ABRAMOWITZ, M., STEGUN, I. A. Handbook of Mathematical Functions. Mineola (USA, NY): Dover Publications, 1968. ISBN: 9780486612720

[18] FRANCESCHETTI, G., TATOIAN, J., GIBBS, G. Timed arrays in a nutshell. IEEE Transactions on Antennas and Propagation, 2005, vol. 53, no. 12, p. 4073-4082. DOI: 10.1109/TAP.2005.859765

\section{About the Authors...}

Ioan E. LAGER was born in 1962. He received his engineer degree (M.Sc.) from the University of Braşov, Romania, and Ph.D. degrees from Delft University of Technology, Netherlands (1996) and from the University of Braşov (1998). His research focuses on applied electromagnetics, especially on time-domain phenomena, and nonuniform array antennas.

Martin ŠTUMPF received his Ph.D.degree in Electrical Engineering from the Brno University of Technology (BUT), Brno, Czech Republic, in 2011. After his Ph.D. research, he spent a year and a half as a Post-Doctoral Fellow with the ESAT-TELEMIC Division, Katholieke Universiteit Leuven, Leuven, Belgium. He is currently an Associate Professor with the Department of Radioelectronics, BUT. During a three-month period in 2018, he was a Visiting Professor at the UAq EMC Laboratory, University of L'Aquila, Italy. He has authored the books "Electromagnetic Reciprocity in Antenna Theory" (Wiley-IEEE Press, 2017), "Pulsed EM Field Computation in Planar Circuits: The Contour Integral Method" (CRC Press, 2018), and "Time-Domain Electromagnetic Reciprocity in Antenna Modeling" (Wiley-IEEE Press, 2019). His main research interests include modeling of electromagnetic wave phenomena with an emphasis on EMC and antenna engineering. 